\title{
QoE Comparison of AL-FEC Algorithms on H.265/HEVC Video and Audio Transmission with MMT
}

\author{
Toshiro Nunome ${ }^{1+}$ and Koki Makino ${ }^{2}$ \\ ${ }^{1}$ Department of Computer Science, Graduate School of Engineering, Nagoya Institute of Technology, Japan \\ ${ }^{2}$ Department of Computer Science, Faculty of Engineering, Nagoya Institute of Technology, Nagoya 466- \\ 8555, Japan
}

\begin{abstract}
This study assesses QoE of H.265/HEVC video and audio IP transmission over MMT with the two AL-FEC coding methods. We employ Reed-Solomon and Structured Low Density Parity Check. We perform multidimensional QoE assessment with three adjective pairs. We then find the appropriate selection of the coding method and the code rate according to the content and network condition can enhance QoE.
\end{abstract}

Keywords: MMT, streaming, QoE, AL-FEC, RS, S-LDPC

\section{Introduction}

MPEG has standardized MMT (MPEG Media Transport) [1] as an application-level media streaming protocol not only video but also various media types. It is standardized for replacing MPEG2-TS, which has been widely used in broadcasting, and for considering IP transmission. In the best-effort IP networks, packet loss and delay fluctuation occur, and then the output quality of media streaming can degrade. In MMT, we can utilize AL-FEC (Application Level Forward Error Correction) for recovering from errors and packet losses over the networks.

For network services, QoE (Quality of Experience) [2] is the ultimate quality metric. In [3], Nunome evaluates QoE of audio and video transmission with AL-FEC over MMT. The study does not utilize ALFEC. Reference [4] introduces AL-FEC for QoE assessment of H.264/AVC video and audio transmission by means of MMT. The study employs Reed-Solomon as a coding algorithm. On the other hand, MMT has six candidates for coding algorithms [5].

Thus, this paper assesses QoE with two types of AL-FEC coding algorithms. We employ Reed-Solomon (RS) and Structured Low Density Parity Check (S-LDPC). S-LDPC has higher efficiency than RS for large data blocks. We then show the effect of the algorithms from a QoE point of view.

The remainder of this paper is organized as follows. Section 2 describes the experimental system and the QoE assessment method. Section 3 presents experimental results. Section 4 concludes this paper.

\section{Experimental System}

Figure 1 shows the experimental system. All the links in the network are $100 \mathrm{Mbps}$ full-duplex Ethernet. Media Server transmits video and audio streams to Media Client through MMTP (MPEG Media Transport Protocol); it is an application-level protocol for multimedia transmission [1]. UDP is employed as the transport protocol under MMTP.

\footnotetext{
${ }^{+}$Corresponding author. Tel.: +81-52-735-7785; fax: +81-52-735-5442.

E-mail address: nunome@nitech.ac.jp.
} 


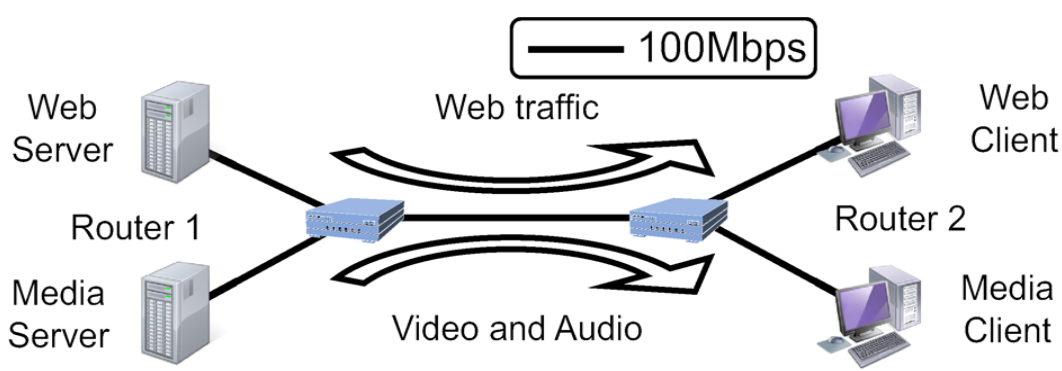

Fig. 1: Experimental network.

In audio, each MMTP/UDP packet includes an MU (Media Unit), which is an information unit for media synchronization control. For simplicity of implementation, we do not employ AL-FEC for audio.

As for the video, we consider a slice as a source packet for AL-FEC. The divided blocks from the slice are called source code blocks. From the blocks, the FEC code generation mechanism generates repair blocks. If the number of source code blocks is not enough to generate FEC repair blocks, we combine several slices and generate source code blocks from the combined slices. The size of the source code block in this paper is 512 bytes. This study is the first step; we then need to evaluate the effect of the source code block size in a future study.

We employ H.265/HEVC video $(1920 \times 1080$ pixels) and AAC-LC (Advanced Audio Coding-Low Complexity) CBR (Constant BitRate) stereo audio. We utilize $\mathrm{x} 265$ as a video encoder. The video encoding bitrate is set to about 3 Mbps or 6 Mbps. We consider a video frame as a video MU. The MU rate is 29.97 MU/s. We deal with the picture pattern IPPPP (I+4P's) and the four slices per frame. The average bitrate and the MU rate of audio are $128 \mathrm{kbps}$ and $46.875 \mathrm{MU} / \mathrm{s}$, respectively. We employ two contents: drama (a scene of historical drama) and sport (a scene of figureskating). Both contents have BGM and scene changes, and sport has a larger movement than drama. The duration is 20 seconds.

Media Client outputs received audio and video after simple playout buffering control. We set the playout buffering time to $500 \mathrm{~ms}$. In Media Client, we utilize FFmpeg for video decoding. Media Client does not use any error concealment techniques for video output.

As the interference traffic of audio and video, Web Server transmits Web traffic to Web Client according to requests generated by WebStone 2.5, which is a Web server benchmark tool. For the number of client processes, we employ 10 and 20.

To implement AL-FEC, we employ OpenFEC, which is an open-source library of FEC. We utilize ReedSolomon and S-LDPC for the FEC algorithms. As the values of the code rate of FEC, we use 1/2, 2/3, and 5/6. We compare them with a method which does not perform FEC code generation (i.e., the code rate is 1). As the total video bitrate of the source video stream and FEC blocks, we consider two values: 3 Mbps and 6 Mbps. When we do not employ FEC, we use 3 Mbps or 6 Mbps as the video encoding bitrate. For employing FEC, we set the video encoding bitrate with consideration of the code rate: $1.5 \mathrm{Mbps}$ and $3 \mathrm{Mbps}$ for the code rate 1/2, $2 \mathrm{Mbps}$ and $4 \mathrm{Mbps}$ for the code rate 2/3, and $2.5 \mathrm{Mbps}$ and $5 \mathrm{Mbps}$ for the code rate 5/6.

For the QoE assessment, we perform a subjective experiment. In the experiment, we ask the assessors to evaluate video and audio output at Media Client. To reproduce the experimental conditions easily, we employ trace files which record the receive timing of video and audio MMTP packets. The assessors evaluate the first 10 seconds of the video and audio transmission.

The assessors are 15 male students of our university who major in computer science. We perform multidimensional QoE assessment with three adjective pairs shown in Table 1. Each adjective pair is scored within five grades. Score 5 means the right-side adjective of each pair, and score 1 represents the left-side one. Score 3 expresses moderate. We then calculate MOS (Mean Opinion Score) by averaging all the assessors' score for each adjective pair. 
Table 1: Adjective pairs

\begin{tabular}{|l|l|}
\hline item & adjective pair \\
\hline video resolution & video is jaggy - clear \\
\hline video collapse & video is corrupt - neat \\
\hline overall quality & $\begin{array}{l}\text { overall quality of video and audio } \\
\text { is bad }- \text { excellent }\end{array}$ \\
\hline
\end{tabular}

\section{Experimental Results}

Before the consideration of QoE metrics, we mention the application-level QoS assessment. The application-level QoS is closely related to QoE because the application-level QoS is adjacent to QoE in the hierarchical network structure. We have evaluated the MU loss ratio (ratio of the number of MUs not output to the number of transmitted MUs), the slice loss ratio of output video (percentage of lost slices in an output frame), and the PSNR (Peak Signal-to-Noise Ratio) of output video. We then found that the application-level QoS is not largely affected by the AL-FEC coding algorithms. Hence, we focus on the QoE assessment results in this paper.

Figure 2 depicts the MOS of "video is jaggy - clear," which represents the sharpness of the image. The result of "video is corrupt - neat" is shown in Fig. 3; it is a measure for blockiness. Figures 2 and 3 are the results for the video bitrate $3 \mathrm{Mbps}$. We present the results of "overall quality of video and audio is bad excellent" in Fig. 4 for the video bitrate 3 Mbps and in Fig. 5 for the video bitrate 6 Mbps. The figures also show the $95 \%$ confidence intervals.

Video encoding bitrate $/$ code rate $=3 \mathrm{Mbps}$

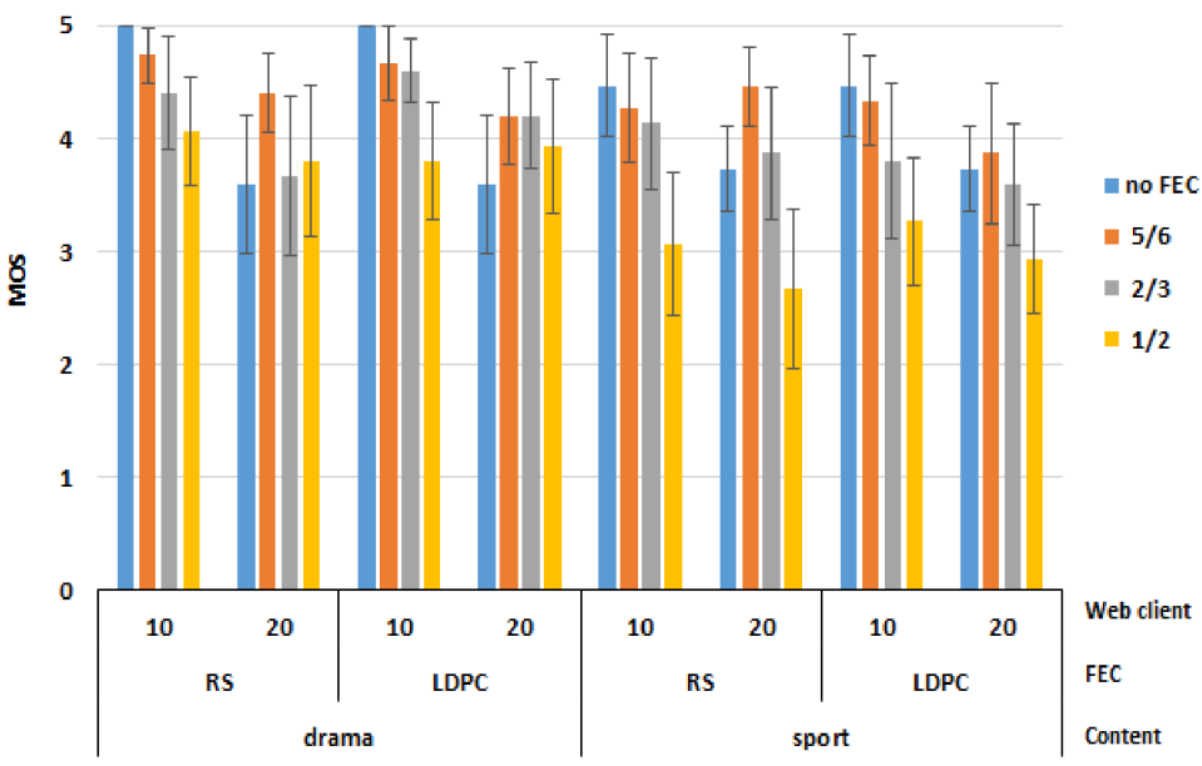

Fig. 2: MOS of "video is jaggy - clear" (video bitrate 3 Mbps).

We find in Fig. 2 that the methods without FEC have larger MOS values of "video is jaggy - clear" than those with FEC under the lightly loaded condition (10 Web clients). The main reason is the higher encoding bit rate in the methods without FEC. The higher encoding bit rate can provide better video image quality. On the other hand, the code rate 5/6 has the highest MOS value under the heavily loaded condition (20 Web clients). The corruption of video output makes the users difficult to notice a slight quality difference between $5 / 6$ and without FEC, and then the effect of corruption becomes dominant for the users' quality perception. 


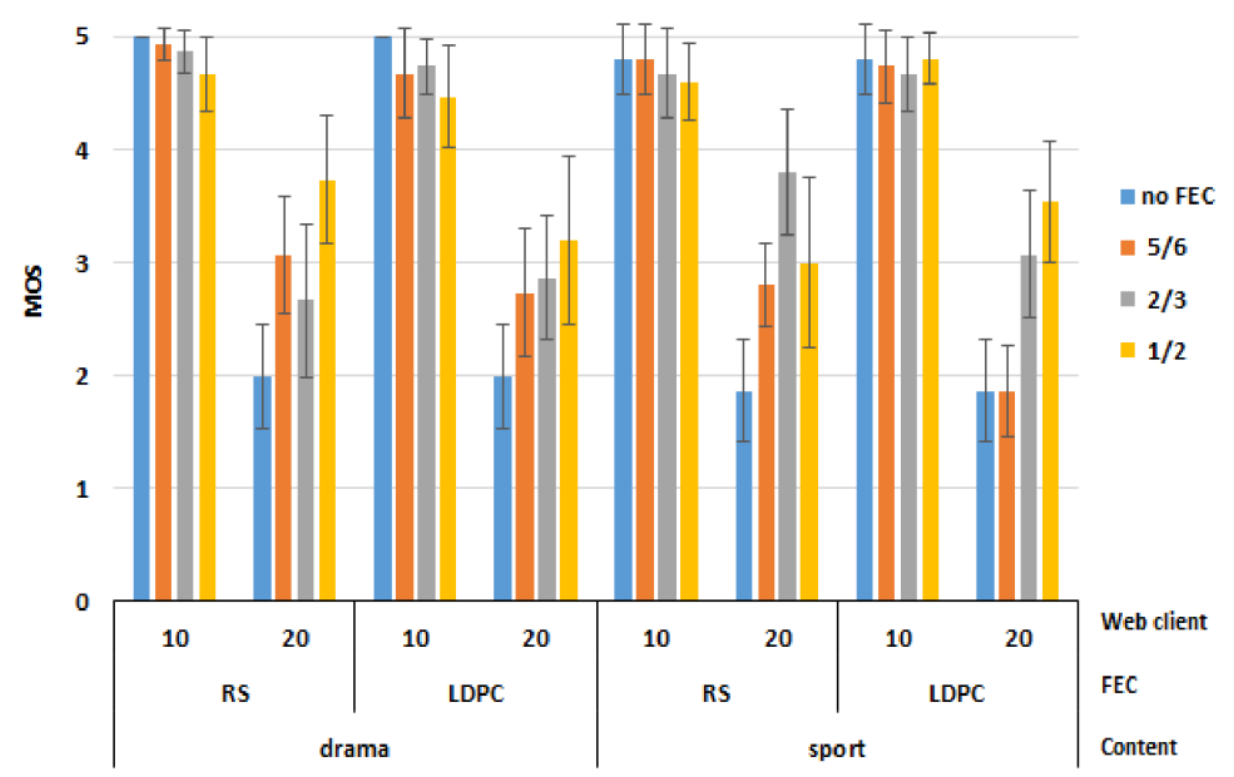

Fig. 3: MOS of "video is corrupt - neat" (video bitrate 3 Mbps).

Video encoding bitrate $/$ code rate $=3 \mathrm{Mbps}$

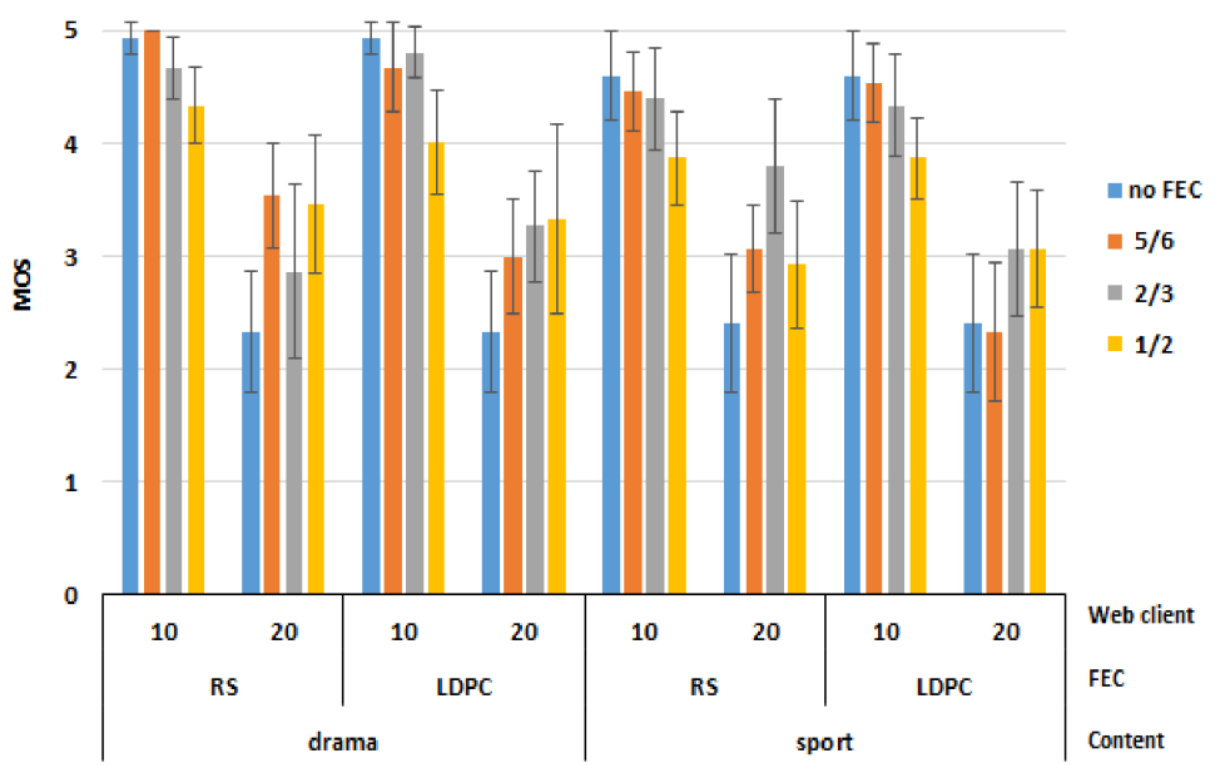

Fig. 4: MOS of "overall quality of video and audio is bad - excellent" (video bitrate $3 \mathrm{Mbps).}$

In Fig. 3, we hardly find the difference among the code rate values and the coding methods under the lightly loaded condition. This is because of the small loss ratio of video packets. On the other hand, on the heavily loaded condition, the MOS value of "video is corrupt - neat" is correlated with the code rate. The larger redundancy with the lower code rate can provide higher MOS value. For sport, the code rate $2 / 3$ has the highest MOS value for RS, and the code rate $1 / 2$ is the best for S-LDPC; i.e., the best code rate is different for the coding methods.

For the video bitrate $3 \mathrm{Mbps}$, we notice in Fig. 4 that the code rates 5/6 and 1/2 have the highest MOS values for RS and S-LDPC, respectively, for drama under the heavily loaded condition. Meanwhile, the code rate $2 / 3$ is better for sport. This implies that a tradeoff relationship between FEC redundancy and encoding bitrate is affected by the contents and the coding algorithms. 


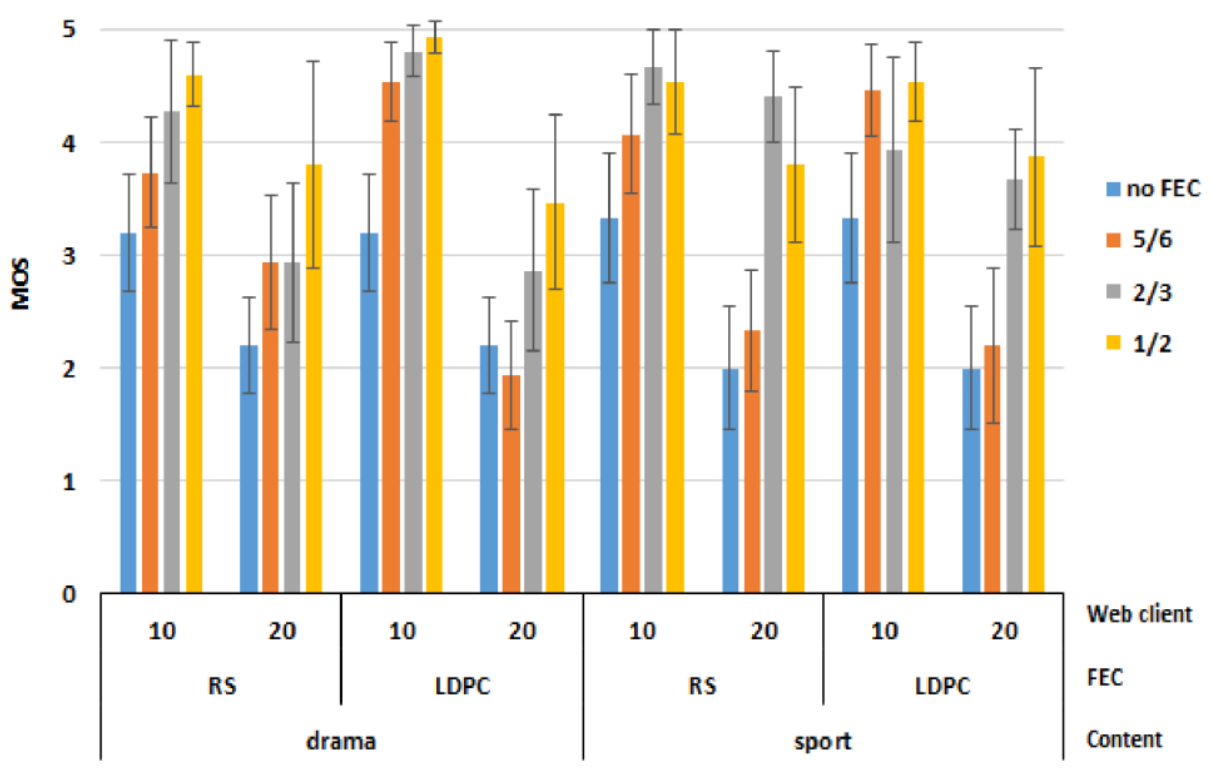

Fig. 5: MOS of "overall quality of video and audio is bad - excellent" (video bitrate $6 \mathrm{Mbps}$ )

In Fig. 5, when the video bitrate is 6 Mbps, we see that the higher redundancy (lower code rate) has higher MOS value of "overall quality of video and audio is bad - excellent" for drama. This is because even the most redundant situation in this paper (i.e., the code rate 1/2), the video encoding bitrate is $3 \mathrm{Mbps}$; it can provide enough video quality.

\section{Conclusions}

In this study, we assessed the QoE of H.265/HEVC video and audio IP transmission over MMT with the two AL-FEC coding methods. We then found that the appropriate selection of the coding method and the code rate according to the content and network condition can enhance QoE.

In future work, we need to assess the effect of AL-FEC coding mechanisms under various conditions including wireless networks. We will also perform an evaluation with FEC mechanisms for audio.

\section{References}

[1] ITU-T Rec. P.10/G.100, "Vocabulary for performance, quality of service and quality of experience," Nov. 2017.

[2] ISO/IEC 23008-1, "Information technology - High efficiency coding and media delivery in heterogeneous environments - Part 1: MPEG media transport (MMT)," Second edition, Aug. 2017.

[3] T. Nunome, "A video output method for H.265/HEVC video and audio IP transmission and its QoE," Proc. 25th International Conference on Telecommunications (ICT 2018), pp. 259-263, June 2018.

[4] T. Nunome, "The joint effect of MMT AL-FEC and error concealment on video streaming QoE, " Proc. IEEE International Conference on Cloud Networking (CloudNet 2018), Oct. 2018.

[5] ISO/IEC 23008-10, "Information technology - High efficiency coding and media delivery in heterogeneous environments - Part 10: MPEG media transport forward error correction (FEC) codes,” Apr. 2015. 
